\title{
New data on Pisaura novicia (Aranei: Pisauridae) from eastern Europe
}

\author{
Новые данные о Pisaura novicia (Aranei: Pisauridae) \\ из Восточной Европы
}

\author{
Anton A. Nadolny ${ }^{1}$, Alexandr V. Ponomarev ${ }^{2}$, Mykola M. Kovblyuk ${ }^{1}$, \\ Konstantin V. Dvadnenko \\ А.А. Надольный ${ }^{1}$, А.В. Пономарёв ${ }^{2}$, Н.М. Ковблюк ${ }^{1}$, К.В. Авадненко \\ ${ }^{1}$ Zoology Department, V.I. Vernadsky Taurida National University, Yaltinskaya str. 4, Simferopol 95007, Ukraine. E-mail: \\ nadolnyanton@mail.ru; kovblyuk@mail.ru \\ ${ }^{2}$ Institute of Arid Zones, South Scientific Centre RAS, Chekhov str. 41, Rostov-on-Don 344006, Russia. E-mail: ponomarev1952@mail.ru \\ ${ }^{1}$ Кафедра зоологии Таврического национального университета им. В.И. Вернадского, ул. Ялтинская 4, Симферополь 95007 , \\ Украина. \\ ${ }^{2}$ Институт аридных зон ЮНЦ РАН, Южный научный центр РАН, пр. Чехова 41, Ростов-на-Дону 344006, Россия.
}

KEY WORDS: Pisauridae, Pisaura, new records, redescriptions, spatial distribution, phenology.

КЛЮЧЕВЫЕ СЛОВА: Pisauridae, Pisaura, новые находки, переописания, ландшафтное распределение, фенология.

ABSTRACT. Pisaura novicia (L. Koch, 1878) is found in Ukraine for the first time; it is also recorded from the southeastern regions of European Russia and Abkhazia. Illustrated redescriptions and diagnoses of $P$. novicia and P. mirabilis (Clerck, 1757) are provided. Spatial distribution and seasonal dynamics of activity of both species in the Crimea are given.

РЕЗЮМЕ. На Украине, в южных регионах европейской части России и Абхазии отмечен вид Pisaura novicia (L. Koch, 1878), новый для фауны Украины. Представлены иллюстрированные переописания и диагнозы P. novicia и P. mirabilis (Clerck, 1757). Для обоих видов приведены сведения о распределении по ландшафтным зонам и фенологии в Крыму.

\section{Introduction}

The genus Pisaura Simon, 1885, contains 13 species distributed in the Palaearctic Region, with P. mirabilis (Clerck, 1757) being the type species [Platnick, 2012]. It is the only widespread species known from the West-Central Palaearctics [Levy, 1999; Zhang, Zhu \& Song, 2004; Helsdingen, 2011]. Other species have local ranges and are known from Macaronesia, the Mediterranean and east Asia. Six species are known from Macaronesia and the Mediterranean: P. acoreensis Wunderlich, 1992; P. consocia (O. Pickard-Cambridge, 1872); P. mirabilis; P. novicia; P. orientalis Kulczyński, 1913, and P. quadrilineata (Lucas, 1838). Six species are known from east Asia: $P$. anahitiformis Kishida, 1910; P. ancora Paik, 1969; P. bicornis Zhang et Song, 1992; P. lama Bösenberg et Strand, 1906; P. mirabilis and $P$. sublama Zhang, 2000. In addition, $P$. podilensis Patel et Reddy, 1990, and P. swamii Patel, 1987, have been described and are known from India [Patel, 1987; Patel \& Reddy, 1990]. Thus, Pisaura has two centers of species diversity: the Mediterranean and east Asia. Most of the European species are well known, except for $P$. novicia and $P$. orientalis, which to date were known from old descriptions only.

Two species of Pisaura have been recorded from the Caucasus [Otto \& Dietzold, 2006]: the well-known $P$. mirabilis and poorly known $P$. novicia. While studying the spiders collected from Abkhazia we found a few Pisaura females, which clearly differed from those of $P$. mirabilis [see Kovblyuk et al., 2011], and which were provisionally assigned to $P$. novicia. This record helped us to find more males and females of $P$. novicia in the spider collections from the Crimea, southeastern regions of European Russia and the Caucasus. This species was originally described from Borzhomi and Tuapse, with the drawings of male palp and tibial apophysis [see L. Koch, 1878: tab. II, f. 3, 3a, 3b]. The males of $P$. novicia from our material have similar shape of the tibial apophysis (cf. Figs 20-21).

This paper presents illustrated redescriptions both of $P$. mirabilis and of $P$. novicia, and rectifies their distribution.

\section{Material and Methods}

All specimens used for this study are deposited in the private collection of A.V. Ponomarev (CP) and the National Arachnological Collection, Zoology Depart- 

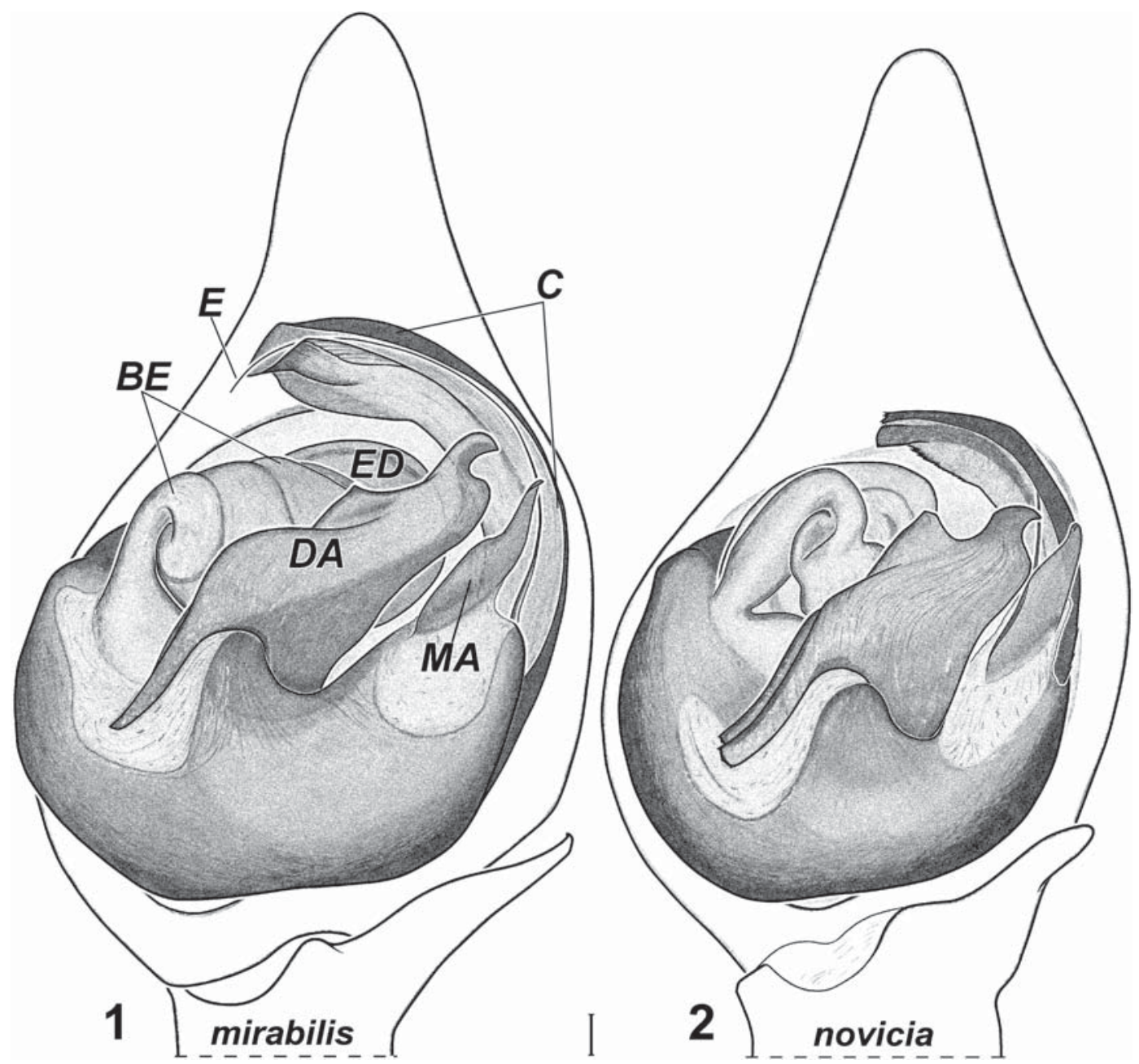

Figs 1-2. Male palps of Pisaura mirabilis (1) and P. novicia (2) from the Crimea, ventral view. Scale bar: $0.1 \mathrm{~mm}$. Abbreviations: $B E-$ base of embolic division; $C$ - conductor; $D A$ - distal apophysis; $E$ - embolus; $E D$ - embolic division; $M A$ - median apophysis.

Рис. 1-2. Пальпы самцов Pisaura mirabilis (1) и P. novicia (2) из Крыма, вентрально. Масштаб 0,1 мм. Обозначения: $B E-$ основа эмболярного отдела; $C$ - кондуктор; $D A$ - дистальный отросток; $E$ - эмболюс; $E D$ - эмболярный отдел; $M A-$ медиальный отросток.

ment, V.I. Vernadsky Taurida National University, Simferopol, Ukraine, curator of collection M.M. Kovblyuk (TNU)

Illustrations were made using reflecting and transmitted light microscopes. Illustrations of the epigynes were made after maceration in $20 \% \mathrm{KOH}$ solution. Scanning electron photos were taken using the EVO40 XVP (LEO143OVP) scanning microscope in the Interdisciplinary Laboratory, Institute of Arid Zones, South Scientific Centre RAS, Rostov-on-Don. Leg and palp segments were measured after their separation from the cephalothorax. All measurements are in millimeters.

Terminology used for the description of copulatory organs and abbreviations used in figures follows Sierwald [1989] and Zhang et al. [2004] (with modifications, e.g., $E D$ and $\operatorname{Tr} A$ ): $A E P$ - anterior epigynal pocket (or small epigynal pit); $B E$ - base of embolic division; $C$ - conductor; $C F-$ copulatory fold; $C O-$ copulatory opening; $D A-$ distal apophysis; $E-$ em- bolus; $E D$ - embolic division; $F D$ - fertilization duct; $H S$ - head of spermathecae; $L L$ - lateral lobe; $M A$ - median apophysis; $S$ - septum; $\operatorname{Tr} A$ - terminal apophysis. The following abbreviations are used in the text: a - apical; d - dorsal; pl - prolateral; $\mathrm{rl}$ retrolateral; v - ventral. Terminology for distribution patterns follows the system proposed by K.B. Gorodkov [1984].

\section{Species survey}

Pisaura mirabilis (Clerck, 1757)

Figs 1, 3, 5, 7, 9-11, 15-16, 19, 22-23, 26-27, 31$32,35,39$.

For a complete list of references see Platnick [2012].

MATERIAL EXAMINED. RUSSIA. Adygeya Republic: Maykopskiy Distr.: $1 \sigma^{\top}, 1$ ( $(\mathrm{CP})$, nr. Dakhovskaya Vil., Lago-Naki Mt., meadow, 24.06.2011, D.D. Volkova. Astrakhan' Area: Astrakhan' Distr.: 1 O', 5 우 (CP), Bogdinsko-Baskunchakskiy Reserve, 7-10.06.2005, E.A. Belosludtsev \& A.S. Tili. Krasnyi Yar Distr.: 
mirabilis
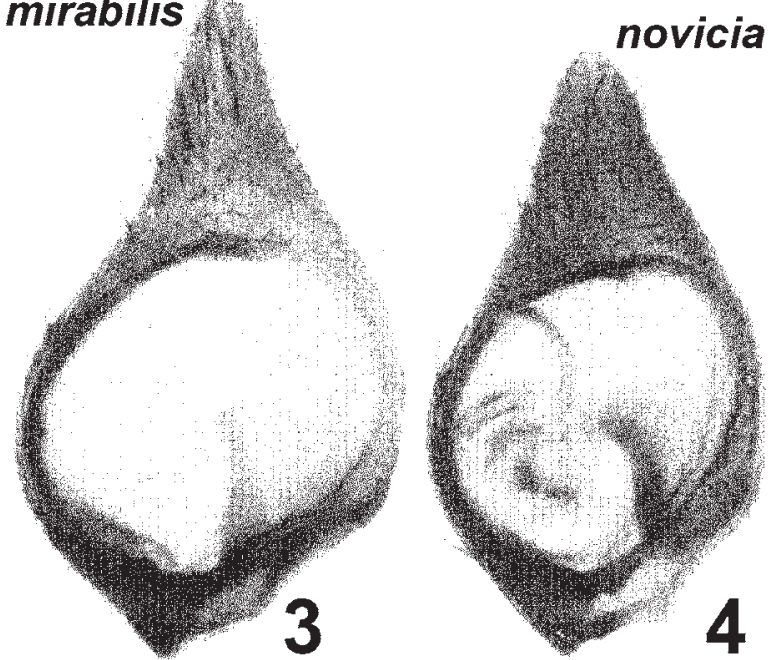

Figs 3-4. Cymbium of Pisaura mirabilis (3) and $P$. novicia (4) from the Crimea, ventral view.

Рис. 3-4. Цимбиумы Pisaura mirabilis (3) и P. novicia (4) из Крыма, вентрально.

1 (CP), Dosang, 17.06.2006, A.S. Matyukhin. Dagestan Republic: Kizlyar Distr.: 1 (CP), Tyuleniy Island, pitfall traps, 22 26.06.2011, S.V. Alieva. North Osetiya Republic: Ardon Distr.: 1 ㅇ (CP), $1 \mathrm{~km} \mathrm{~S}$ Bekan Vil., Bekan water reservoir, $530 \mathrm{~m}$, 21.07.2011, Yu.E. Komarov. Rostov Area: Azov Distr.: 2 우 (CP), Don River delta, Donskoy Vil., meadow, 28.06.2012, 12.07.2012, A.V. Ponomarev. Ust-Donetsk Distr.: $10 \sigma^{7} \sigma^{7}, 1$ ㅇ (CP), Razdorskaya Vil., Atamanskaya Valley, woodland plantation, 17.0519.07.2001, A.V. Ponomarev; $2 \sigma^{7} \sigma^{7}$ (CP), Krimskiy Vil., Savateevskaya Valley, pitfall traps, 15.05-3.06.2006, A.V. Ponomarev. Orlovskiy Distr.: $1 \sigma^{\top}$ (CP), Kamyshevka Vil., 22.06.2011, A.V. Ponomarev. Ul'yanovsk Area: Ul'yanovsk Distr.: 1 ㅇ (CP), Ul'yanovsk, 06.2009, A.A. Zotov \& I. Strakhova. Sengiley Distr. 3 우 (CP), vicinity of Artyushkino Vil., 10-16.07.2009, V.P. Isaev UKRAINE. The Crimea: Bakhchisaray Distr.: 1 (TNU-1201/1/ 1), Crimean State Nature Reserve, ca. 2 km NW kordon Zelenyi Gai, bank of Alma River, 15.06.2000, M.M. Kovblyuk. Chernomorskoe Distr.: $3 \sigma^{7} \sigma^{7}, 8$ 우, 5 cocoons (TNU), vicinity of Chernomorskoe, Kipchak Valley and Dzhangul' Coast, 29.05-2.06.2012, A.A. Nadolny. Dzhankoy Distr.: $1 \sigma^{7}$ (TNU-SO68), $6.5 \mathrm{~km}$ N Solenoe Ozero Vil., N45 $56^{\prime} 35^{\prime \prime}$ E34 $4^{\circ} 27^{\prime} 06.7^{\prime \prime}$, Phragmites \& Carex on mollusk shells bar, pitfall traps, 8-29.05.2009, A.A. Nadolny. Feodosiya Distr.: 20 O $^{\top} \sigma^{7}, 13$ 우 (TNU-1760/2, 1776/1, 1972/1/1, 2039/ $1 / 1,2041 / 1,2043 / 1,2311 / 1,2378 / 1,2380 / 1,2383 / 1 / 1,2562 / 1$, $2593 / 1 / 1,2600 / 4,2610 / 1 / 1,2611 / 1,2612 / 1,2737 / 5,2774 / 1,2775 /$ 1, 2867/1, 2869/1), Karadag Nature Reserve, by hand picking and pitfall traps, 2.05.2003-20.06.2008, M.M. Kovblyuk, O.V. Kukushkin, A.A. Nadolny. Saky Distr.: $6 \sigma^{7} \sigma^{7}, 3$ 什 (TNU-1654/1, 1682/2, 1702/4, 1703/1), vicinity of Pribrezhnaya railway station, pitfall traps, 30.04-3.07.2000, M.M. Kovblyuk. Sevastopol Distr.: 2 우 (TNU), Sevastopol, Khersones ancient town, 19.06.1998, M.M Kovblyuk; 1 (TNU), Sevastopol, Sarandinakina ravine, 11.06.2000, O.V. Kukushkin. Simferopol Distr.: 5 + (TNU), Lozovoe Vil., nr. Simferopol water reservoir, 25.04.1996, V. Zykova; $6 \sigma^{7} \sigma^{7}, 3$ of (TNU-1757/1, 1836/1, 1845/1, 1857/1, 1858/1), vicinity of Skvortsovo Vil., pitfall traps, 9.05-9.06.2002, M.M. Kovblyuk. Sudak Distr.: $4 \sigma^{7} \sigma^{7}, 1$ ( (TNU-2691/1, 2696/1, 2697/1, 2707/1), ca. $10 \mathrm{~km} \mathrm{~W}$ Sudak, vicinity of Mezhdurech'e Vil., pitfall traps, 23.05-24.06.2010, M.K. Yusufova. Lenin Distr.: 2 우 (TNU), Kerch peninsula, coast between Chagani Cape and Chokrak Lake, 30.06-6.07.1999, O.V. Kukushkin; 15 +o , 15 cocoons (TNU), E bank of Tobechik Lake, extracted out of the nests, 31.05.2007, A.A. Nadolny. Kyiv Area: 2 우 (TNU), Tetiiv, 21.07-2.08.2006, A.A. Nadolny.

DIAGNOSIS. The females of $P$. mirabilis can be easily distinguished from all other congeners by the wide funnel-like copulatory openings (see Figs 26-27, 31,39 ) (other species have the narrow, slit-like copulatory openings). The males of $P$. mirabilis are close to those of $P$. novicia, but can be distinguished by the narrower distal part of tibial apophysis, without curvature, and projection on its anterior side (in P. novicia, the tibial apophysis is curved dorsally and has a small projection on its anterior side; cf. Figs 15-21). The diameter of the embolic division of $P$. mirabilis is 1.3 times larger than that of $P$. novicia (cf. Figs 22-25). The median apophysis and conductor of $P$. mirabilis are longer than those of $P$. novicia (cf. Figs 5-8). Yet, the shape of the distal apophysis is clearly different in the two species (cf. Figs 1-2, 9-14, 35-38).

DESCRIPTION. Male and female from the Crimea. Measurements (male/female): total length 8.8 / 9.5; carapace 4.0 / 4.1 long, 3.2 / 3.2 wide. Length of palp segments (male/female): femur $2.0 / 1.7$, patella 0.8 / 0.8 , tibia $0.5 / 1.0$, tarsus $2.4 / 1.9$. Length of leg segments (male/female):

\begin{tabular}{|l|l|l|l|l|l|}
\hline Leg & Femur & Patella & Tibia & Metatarsus & Tarsus \\
\hline I & $5.0 / 4.9$ & $1.7 / 1.8$ & $5.2 / 4.8$ & $5.0 / 4.5$ & $2.4 / 2.2$ \\
\hline II & $5.2 / 5.2$ & $1.7 / 1.8$ & $5.2 / 5.0$ & $5.1 / 4.7$ & $2.4 / 2.2$ \\
\hline III & $4.4 / 4.5$ & $1.4 / 1.5$ & $3.7 / 3.8$ & $3.9 / 3.9$ & $1.5 / 1.6$ \\
\hline IV & $5.4 / 5.6$ & $1.4 / 1.6$ & $5.1 / 5.2$ & $5.8 / 6.0$ & $2.2 / 2.2$ \\
\hline
\end{tabular}

Male leg spination.

\begin{tabular}{|c|c|c|c|c|}
\hline & Femur & Patella & Tibia & Metatarsus \\
\hline Leg I & $\begin{array}{l}\text { d 1-1-1 (a), } \\
\text { pl 1-1-1, rl } \\
1-1-1-1-1\end{array}$ & d 1 (a) & $\begin{array}{l}\mathrm{d} 1-1, \mathrm{pl} 1- \\
1, \mathrm{rl} 1-1, \mathrm{v} \\
2-2-2-2 \text { (a) }\end{array}$ & $\begin{array}{l}\text { pl 1-1-1 (a), } \\
\text { rl 1-1-1 (a), v } \\
2-2-3 \text { (a) }\end{array}$ \\
\hline Leg II & $\begin{array}{l}\text { d 1-1-1 (a), } \\
\text { pl 1-1-1-1, } \\
\text { rl 1-1-1-1- } \\
1\end{array}$ & d 1 (a) & $\begin{array}{l}\text { d 1-1, pl 1- } \\
1, \text { rl 1-1, v } \\
2-2-2-2 \text { (a) }\end{array}$ & $\begin{array}{l}\text { pl 1-1-1 (a), } \\
\text { rl 1-1-1 (a), v } \\
2-2-3 \text { (a) }\end{array}$ \\
\hline Leg III & $\begin{array}{l}\text { d 1-1-1 (a), } \\
\text { pl 1-1-1-1- } \\
1, \text { rl 1-1-1- } \\
1\end{array}$ & $\mathrm{~d} 1$ & $\begin{array}{l}\text { d 1-1, pl 1- } \\
1, \text { rl 1-1, v } \\
2-2-2 \text { (a) }\end{array}$ & $\begin{array}{l}\text { pl 1-1-1 (a), } \\
\text { rl 1-1-1 (a), v } \\
2-2-3 \text { (a) }\end{array}$ \\
\hline Leg IV & $\begin{array}{l}\text { d 1-1-1 (a), } \\
\text { pl 1-1-1-1, } \\
\text { rl 1-1-1 }\end{array}$ & d 1 (a) & $\begin{array}{l}\text { d 1-1, pl 1- } \\
1, \text { rl 1-1, v } \\
2-2-2 \text { (a) }\end{array}$ & $\begin{array}{l}\text { pl 1-1-1 (a), } \\
\text { rl 1-1-1 (a), v } \\
2-2-3 \text { (a) }\end{array}$ \\
\hline
\end{tabular}

Male palp spination. Femur: d 1-1, pl 1; patella: d 1-1 (a), pl 1; tibia: d 1, pl 2; cymbium: pl 2, v 2.

Female leg spination.

\begin{tabular}{|c|c|c|c|c|}
\hline & Femur & Patella & Tibia & Metatarsus \\
\hline Leg I & $\begin{array}{l}\text { d 1-1-1 (a), } \\
\text { pl 1-1-1-1, } \\
\text { rl 1-1-1-1-1 }\end{array}$ & d 1 (a) & $\begin{array}{l}\mathrm{d} 1-1, \mathrm{pl} 1- \\
1, \mathrm{rl} 1-1, \mathrm{v} \\
2-2-2-2 \text { (a) }\end{array}$ & $\begin{array}{l}\text { pl 1-1-1 (a), } \\
\text { rl 1-1-1 (a), v } \\
2-2-3 \text { (a) }\end{array}$ \\
\hline Leg II & $\begin{array}{l}\text { d 1-1-1 (a), } \\
\text { pl 1-1-1-1- } \\
1, \text { rl 1-1-1- } \\
1-1\end{array}$ & d 1 (a) & $\begin{array}{l}\mathrm{d} 1-1, \mathrm{pl} 1- \\
1, \mathrm{rl} 1-1, \mathrm{v} \\
2-2-2-2 \text { (a) }\end{array}$ & $\begin{array}{l}\text { pl 1-1-1 (a), } \\
\text { rl 1-1-1 (a), v } \\
2-2-3 \text { (a) }\end{array}$ \\
\hline Leg III & $\begin{array}{l}\text { d 1-1-1 (a), } \\
\text { pl 1-1-1-1- } \\
1, \text { rl 1-1-1- } \\
1-1\end{array}$ & $\begin{array}{l}\text { d 1-1 } \\
\text { (a), pl } \\
1\end{array}$ & $\begin{array}{l}\text { d 1-1, pl 1- } \\
1, \text { rl 1-1, v } \\
2-2-2 \text { (a) }\end{array}$ & $\begin{array}{l}\text { pl 1-1-1 (a), } \\
\text { rl 1-1-1 (a), v } \\
2-2-3 \text { (a) }\end{array}$ \\
\hline Leg IV & $\begin{array}{l}\text { d 1-1-1 (a), } \\
\text { pl 1-1-1, rl } \\
1-1\end{array}$ & d 1 (a) & $\begin{array}{l}\text { d 1-1, pl 1- } \\
1, \text { rl 1-1, v } \\
2-2-2 \text { (a) }\end{array}$ & $\begin{array}{l}\text { pl 1-1-1 (a), } \\
\text { rl 1-1-1 (a), } \\
2-1-2-3 \text { (a) }\end{array}$ \\
\hline
\end{tabular}

Female palp spination. Femur: d 1-1 (a), pl 1, rl 1; patella: d 1-1 (a), pl 1; tibia: d 1-1 (a), pl 2-1; tarsus: pl 2-1, rl 2-1, v 2 (a). 


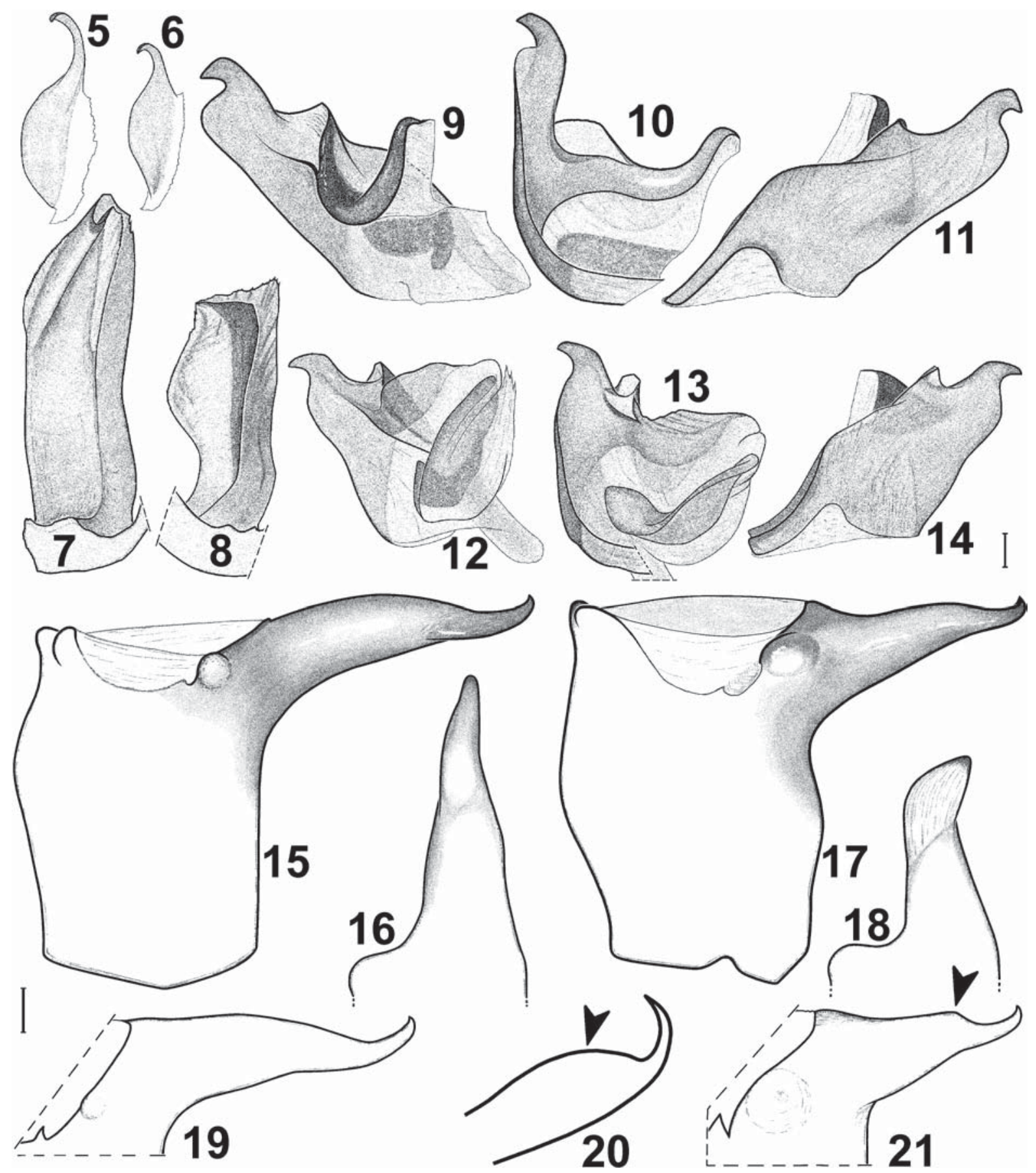

Figs 5-21. Details of the male copulatory organs of Pisaura mirabilis $(5,7,9-11,15-16,19$ [from the Crimea]) and P. novicia $(6,8$, $12-14,17-18,21): 5-6$ - median apophysis, retrolateral view; 7-8 - conductor, rear view; $9-14-$ distal apophysis $(9,12-$ dorsal view; 10,13 - retrolateral view; 11,14 - ventral view); 15,17 - palpal tibia, ventral view; $16,18,19-21$ - tibial apophysis $(16,18$ rear view; 19-21 - ventral view). 20 - after L. Koch [1878]. Scale bar: $0.1 \mathrm{~mm}$.

Рис. 5-21. Части копулятивных органов самцов Pisaura mirabilis $(5,7,9-11,15-16,19$ [из Крыма]) и P. novicia $(6,8,12-14$, 17-18, 20-21): 5-6 - медиальный отросток, ретролатерально; 7-8 - кондуктор, сзади; 9-14 - дистальный отросток $(9,12-$ дорсально; 10, 13 - ретролатерально; 11,14 - вентрально); 15, 17 - голень самца, вентрально; 16, 18, 19-21 - отросток голени (16, 18 - сзади; 19-21 - вентрально). 20 - по L. Koch [1878]. Масштаб 0,1 мм.

Male copulatory organs: Figs 1, 3, 5, 7, 9-11, 15$16,19,22-23,35$. Tegulum has two apophyses on its ventral side (median and distal) and the conductor on its retrolateral side. The median apophysis is a thin transparent plate (Figs 1, 5). The distal apophysis is more massive than the median one and has an appendix on its dorsal side (Figs 1, 9-11,35) attaching it to the base of embolic division (further as $B E$ ). The embolic 


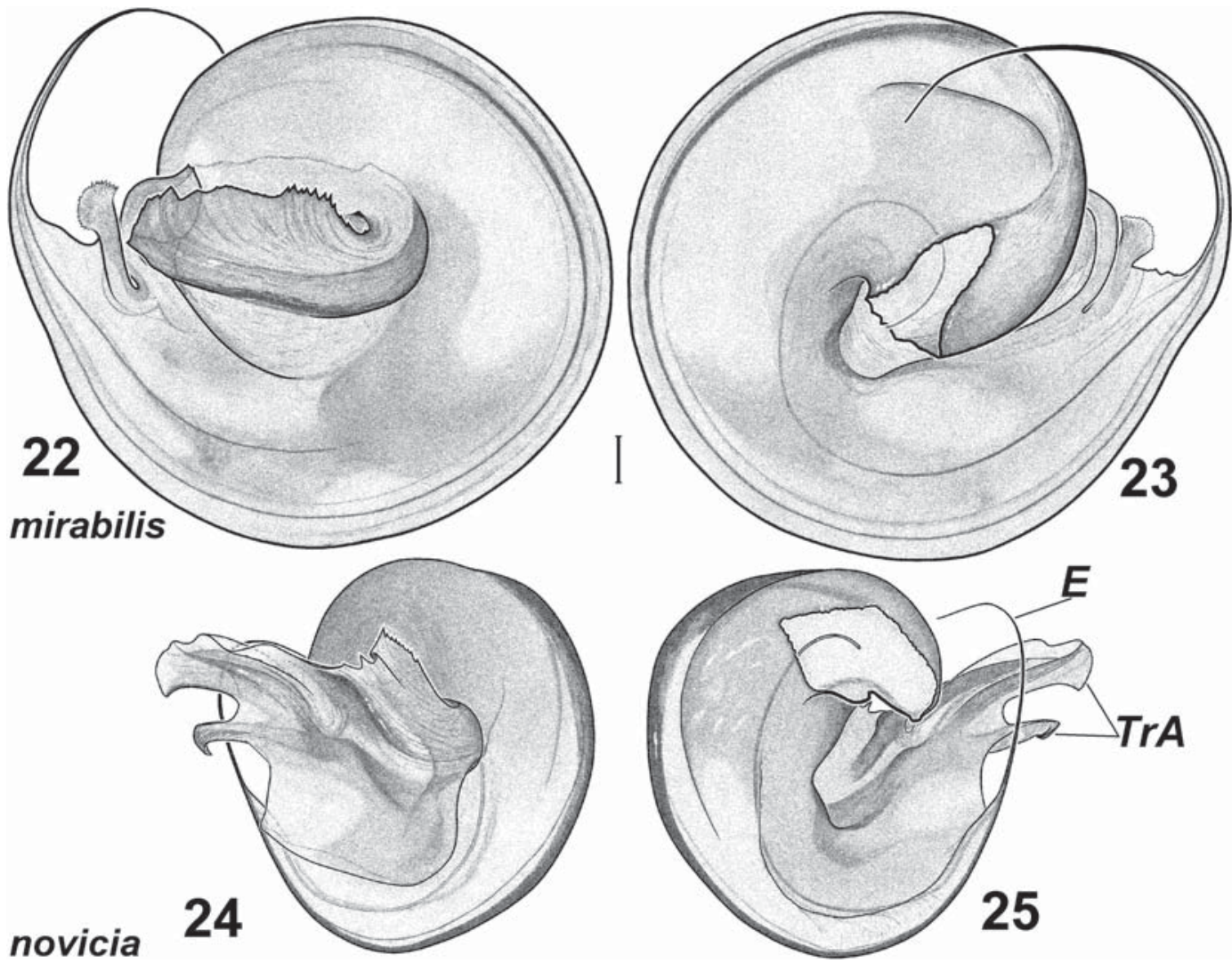

Figs 22-25. Embolic division of Pisaura mirabilis (22-23) and P. novicia (24-25) from the Crimea: 22, 24 - dorsal view; 23, 25 ventral view. Scale bar: $0.1 \mathrm{~mm}$. Abbreviations: $E$ - embolus; $\operatorname{Tr} A-$ terminal apophysis.

Рис. 22-25. Эмболярный отдел Pisaura mirabilis (22-23) и P. novicia (24-25) из Крыма: 22, 24 - дорсально; 23,25 вентрально. Масштаб 0,1 мм. Обозначения: $E$ - эмболюс; $\operatorname{Tr} A$ - терминальный отросток.

division is situated at the distal side of $B E$. The seminal duct passes to the embolic division through $B E$. The embolic division is coiled (Figs 22-23); it continues the spiral-shaped $B E$. The embolic division carries the long embolus and few appendices which are likely to be homologous to the terminal apophysis in the Evippinae [Marusik, Kovblyuk \& Koponen, 2011: fig. 16]. The embolus is situated in the conductor fold (Fig. 1). The subtegulum is situated in the dorsal side of bulbus (normally hidden inside the cymbium).

Female copulatory organs: Figs 26-27, 31-32, 39. The epigyne has a septum, an anterior epigynal pocket and internal structures (ducts, spermathecae) (Figs 3132). The spermathecae and ducts are the invaginations of cuticula. The copulatory openings are connected to the spermathecae by the copulatory fold. The spermathecae are opened into a body cavity by the fertilization ducts. After maceration in $10 \% \mathrm{KOH}$ solution the lateral lobes are expanded, thus the cavity of spermathecae can be seen.

COMMENTS. The invaginations between the septal stem and body coverings were referred to as the copulatory ducts by Zhang et al. [2004: fig. 137]. In our opinion, these ducts are not used for copulation, as they are not connected to the spermathecae, and they opened in the fold near the copulatory openings [see the schematic course of spermathecae duct trajectory given in Kovblyuk et al., 2011: figs 42-43]. The copulation folds, fertilization ducts and spermathecae are formed by the cuticular invagination between the posterior part of septum and lateral lobes.

VARIATION. Male $(\mathrm{n}=5)$ : carapace 4.0-4.4 long, 3.3-3.5 wide; female $(n=5)$ : carapace 3.7-4.6 long, $3.0-3.5$ wide.

TYPE LOCALITY. P. mirabilis was described from Sweden without an exact locality [Clerck, 1757].

DISTRIBUTION. The West-Central Palaearctic temperate-subtropical range: from Portugal in the west (however, the records of $P$. mirabilis from the Canary Islands and Madeira were based on immatures [Wunderlich, 1987] and thus are doubtful), eastward to China (Xinjiang, Tibet, Gansu), northward to Norway and southward to Israel [Levy, 1999; Zhang et al., 2004; Helsdingen, 2011].

PHENOLOGY. In the Crimea, $\sigma^{\top}+$ - IV-VII, the peak of adult activity is in May, females with egg sacs V-VI. Seasonal dynamics of adult activity in the Crimea is shown in Fig. 46. In Britain, $\sigma^{\top} \sigma^{7}-\mathrm{IV}-\mathrm{IX}$, $+0+$ $\mathrm{V}-\mathrm{XI}$, peak in June [Harvey et al., 2002], i.e., a month later than in the Crimea. In Sweden, $\sigma^{\top} \sigma^{T}-$ V-VII, 

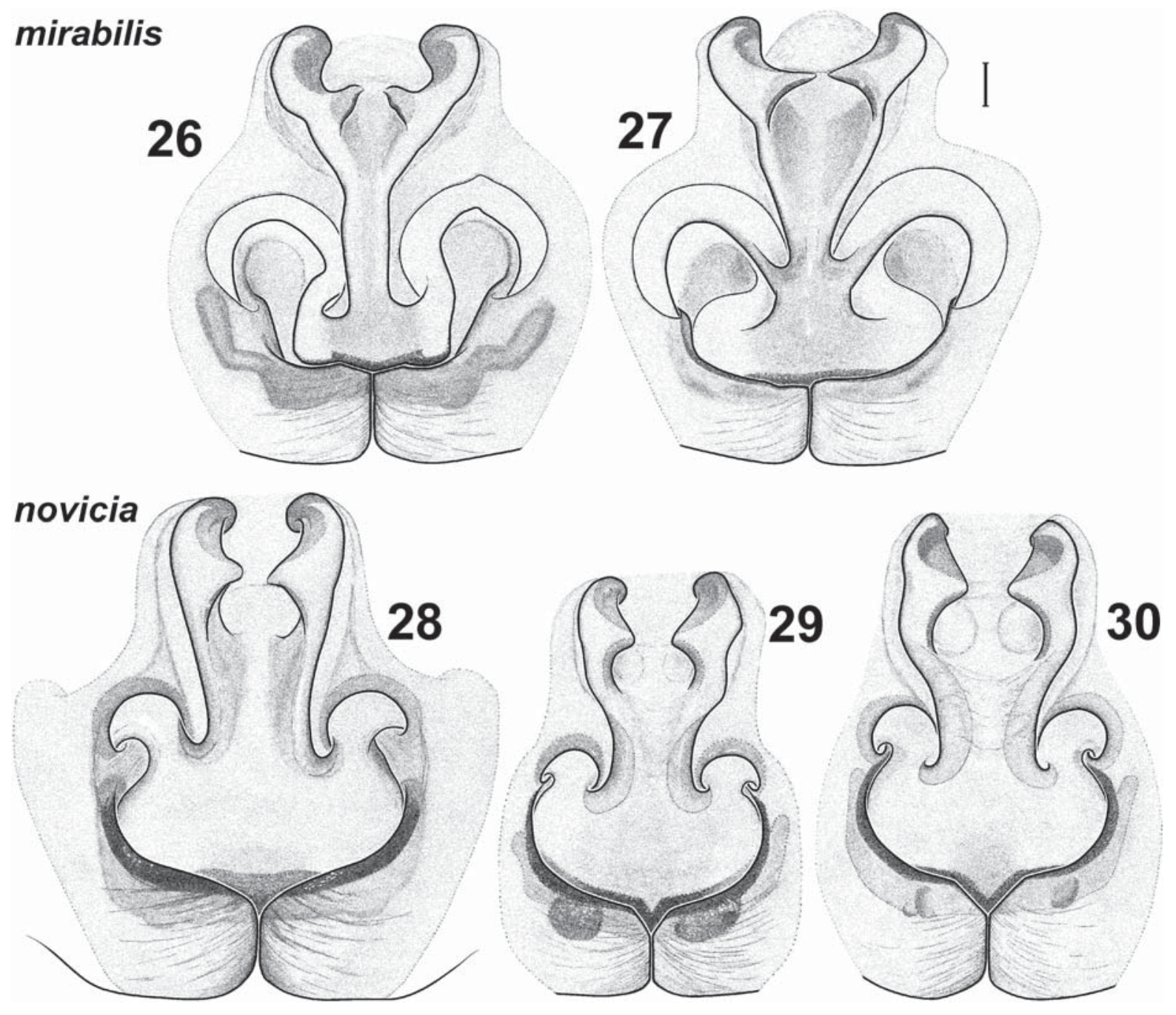

Figs 26-30. Epigynes of Pisaura mirabilis (26-27) and P. novicia (28-30) from the Crimea, ventral view. Scale bar: $0.1 \mathrm{~mm}$. Рис. 26-30. Эпигины Pisaura mirabilis (26-27) и P. novicia (28-30) из Крыма, вентрально. Масштаб 0,1 мм.

O† — VI-IX [Almquist, 2005], i.e., one or two months later than in the Crimea. In Israel, the adult activity begins two months earlier: $\bigcirc^{\top} \sigma^{\top}-$ II-IV,, $0+$ III-V [Levy, 1999].

Pisaura novicia (L. Koch, 1878)

Figs 2, 4, 6, 8, 12-14, 17-18, 20-21, 24-25, 28-30, $33-34,36-38,40-42,43-45$.

Ocyale n. L. Koch, 1878: 54, pl. 2, f. 3, 3a, 3b ( $\left.\sigma^{7}\right)$.

P. mirabilis: Logunov, 1990: 34, pl. 2, f. в, г, д, e, ж, з; pl. 3 , f. II $a$, ІІ $\sigma$ ( $\sigma^{7}$, ; misidentification).

P. n.: Kovblyuk et al., 2011: 38, f. 37-38, 41 ( $(+)$.

Only the references that allow one to identify the species are presented. For a complete list see Platnick [2012].

MATERIAL EXAMINED. ABKHAZIA. Gagra Distr.: 1 (CP13.10.1/9), Gagra, 12.07.2001, Yu.G. Arzanov; 19 (CP-13.10.1/ 14), Bzyb' Vil., 13-15.06.2005, P.P. Ivliev. 1 o' (CP), Bzyb' Vil., 4-10.05.2009, P.P. Ivliev. Gudauta Distr.: 4 q0 (TNU-2636/27), vicinity of Pitsunda, mouth of Ryapsh River, Myusser Distr. of Pitsundo-Myusser Reserve, N43 $09^{\prime} \mathrm{E} 40^{\circ} 25^{\prime}, 27 \mathrm{~m}$, colchis wood
(Arbutus, Alnus, Ficus, Carpinus, Quercus, Acer, Rhododendron, Erica arborea, Rubus, Smilax excelsa, Castanea sativa, Laurocerasus officinalis, Cornus mas, Pinus pithyusa, Crataegus, Tilia, Ruscus), 1-7.07.2008, M.M. Kovblyuk; 2 우 (TNU-2651/1), vicinity of Pitsunda, Myusser Distr. of Pitsundo-Myusser Reserve, left bank of Ryapsh River, N43 ${ }^{\circ} 10^{\prime} \mathrm{E} 40^{\circ} 25^{\prime}, 23 \mathrm{~m}$, colhida wood, sea and river coast, humid gorges, 15-24.07.2009, M.M. Kovblyuk \& N.N. Yunakov. Sukhum Distr.: 19 (TNU-2639/1), Gumysta Reserve, East Gumysta River, kordon Tsymur, N43 $10^{\prime} \mathrm{E} 41^{\circ} 02^{\prime}$, 420 m, wood with Fagus, Acer and Castanea sativa, 8-16.07.2008, M.M. Kovblyuk; 1 (TNU-2644/1), Gumysta Reserve, East Gumysta River, kordon Tsymur, N43 $10^{\prime} \mathrm{E} 41^{\circ} 02^{\prime}, 420 \mathrm{~m}$, wood with Fagus, Acer and Castanea sativa, 26-29.07.2008, M.M. Kovblyuk. RUSSIA. Belgorod Area: Borisovka Distr.: 1 으 (CP), Borisovka Vil., 'Les-na-Vorskle' Reserve, Quercus wood, meadow, 12.06.1996, A.V. Ponomarev; $1 \sigma^{\top}$ (CP), same place, meadow near flood plain of Vorskla River, 3.06.1996, A.V. Ponomarev. Yakovlevskiy Distr.: 1 (CP), $8 \mathrm{~km}$ SE Kustovoe Vil., edge of the damp Salix wood, 7.06.1996, A.V. Ponomarev. Dagestan Republic: $1 \sigma^{7}$ (CP), Makhachkala, Chechen' Island, pitfall traps, 17-21.06.2011, S.V. Alieva. Buynaksk Distr.: 2 우 (CP), Nizhnee Kazanishche Vil., garden, 11-30.06.2008, A.Kh. Khalidov; 3 O $\sigma^{7}$ (CP), Buynaksk, 07.2008, A.Kh. Khalidov; 1 ○', 1 (CP), Novo-Kumukh Vil., 

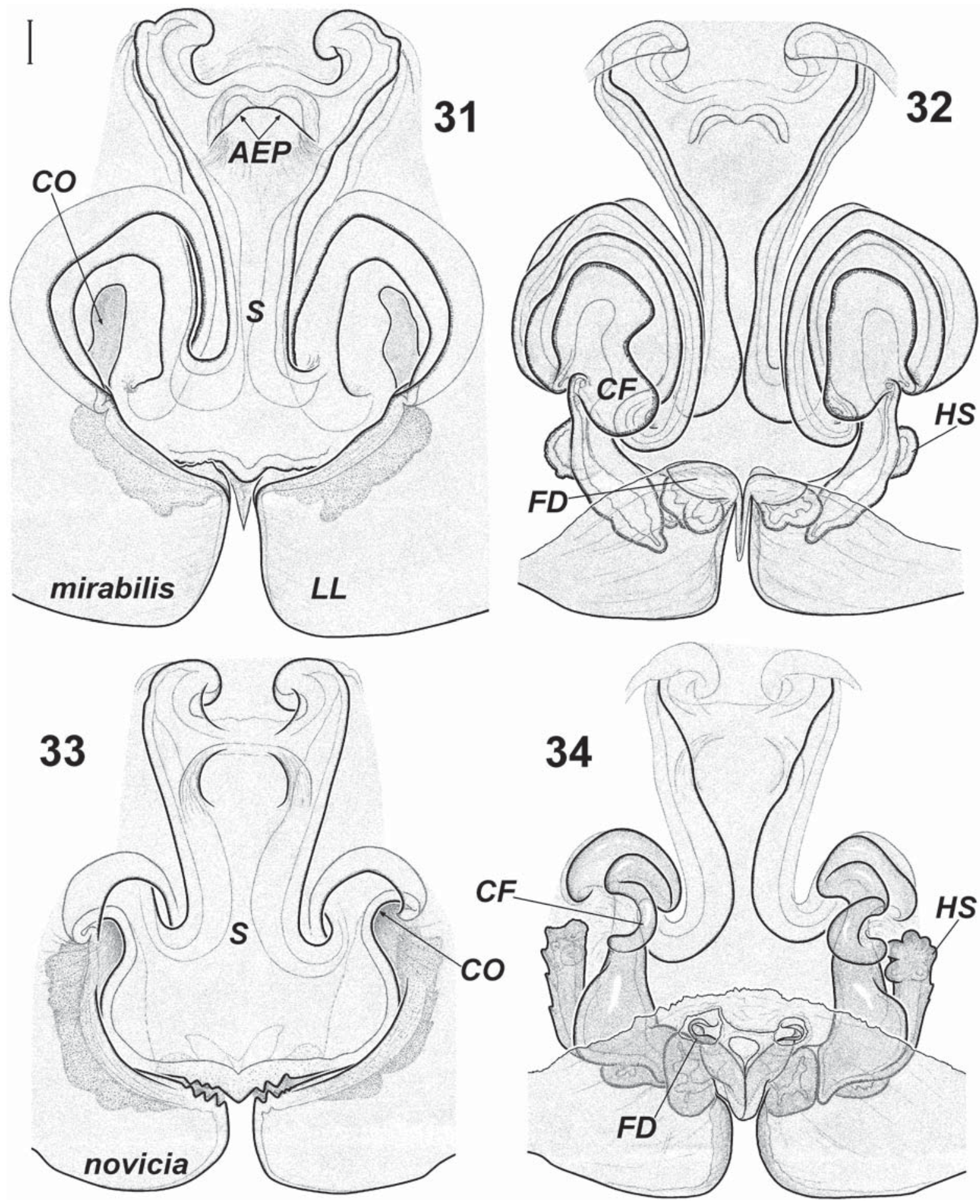

Figs 31-34. Epigyne of Pisaura mirabilis (31-32) and P. novicia (33-34) from the Crimea after maceration in 10\% KOH solution: 31 , 33 - ventral view; 32,34 - dorsal view. Scale bar: $0.1 \mathrm{~mm}$. Abbreviations: $A E P$ — anterior epigynal pocket; $C F$ - copulatory fold; $\mathrm{CO}$ - copulatory opening; $F D$ - fertilization duct; $H S$ - head of spermathecae; $L L$ - lateral lobe; $S$ - septum.

Рис. 31-34. Эпигины Pisaura mirabilis (31-32) и P. novicia (33-34) из Крыма после мацерации в 10\% р-ре КОН: 31 , 33 вентрально; 32, 34 - дорсально. Масштаб 0,1 мм. Обозначения: $A E P$ - передний карман эпигины; $C F$ — копулятивная складка; $C O$ - копулятивное отверстие; $F D$ - оплодотворительный канал; $H S$ — голова сперматеки; $L L-$ боковая пластина; $S$ - септум. 


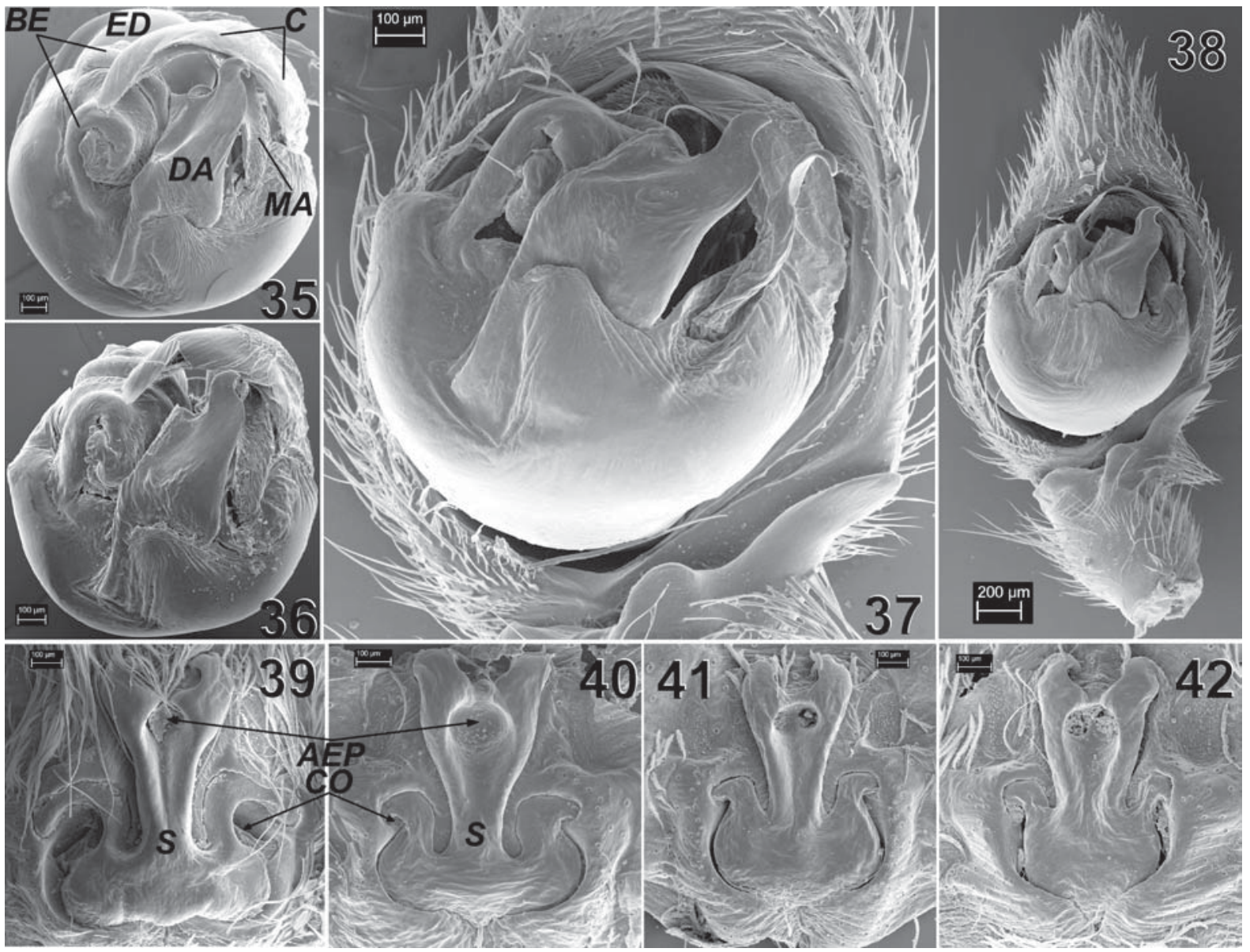

Figs 35-42. Male and female copulatory organs of Pisaura mirabilis (35, 39 - from Rostov Area) and P. novicia (36 - from Karachaevo-Cherkesiya; 38, 42 - from Krasnodar Area; 40 - from Abkhazia; 41 - from Dagestan): 35-36 - bulbus, ventral view; 3738 - male palp, ventral view; 39-42 - epigyne, ventral view. Scale bars: 35-37, 39-42 - 0.1 mm; $38-0.2 \mathrm{~mm}$. Abbreviations: $A E P$ — anterior epigynal pocket; $C O$ - copulatory opening; $B E$ - base of embolic division; $C$ - conductor; $D A$ - distal apophysis; $E D$ embolic division; $M A$ - median apophysis; $S$ - septum.

Рис. 35-42. Копулятивные аппараты самцов и самок Pisaura mirabilis $(35,39$ - из Ростовской обл.) и P. поvicia (36 - из Карачаево-Черкесии; 38, 42 - из Краснодарского края; 40 - из Абхазии; 41 - из Дагестана): 35-36 - бульбус, вентрально; 37 38 - пальпа самца, вентрально; 39-42 - эпигина, вентрально. Масштаб: 35-37, 39-42 - 0,1 мм; 38 - 0,2 мм. Обозначения: $A E P$ - передний карман эпигины; $C O-$ копулятивное отверстие; $B E-$ основа эмболярного отдела; $C$ - кондуктор; $D A-$ дистальный отросток; $E D$ - эмболярный отдел; $M A$ - медиальный отросток; $S$ - септум.

Kara-Kar Valley, garden, 7.06.2009, A.Kh. Khalidov. Gunib Distr.: $1 \mathrm{O}^{7}(\mathrm{CP})$, Verkhniy Gunib Vil., $2000 \mathrm{~m}$ a.s.l., mixed wood on a mountain slope, 07.2008, A.Kh. Khalidov. Karachaevo-Cherkesiya Republic: 1 Oొ7 $^{7} 1$ (CP), Teberda, 07.2010, E.A. Khachikov. Krasnodar Area: 2 우 (CP), vicinity of Krasnodar, woodland plantation, 17.05.2006, V.F. Kobzar'; $1 \mathrm{O}^{7}, 1$ ( $(\mathrm{CP})$, vicinity of Krasnodar, woodland plantation, 7.05.2008, V.F. Kobzar'; 1 \& (CP), Novorossiysk, Shyrokaya Balka Vil., N44ํํ․ $4011^{\prime}$ E $37^{\circ} 42.432^{\prime}$, 28.06.2011, P.P. Ivliev. Anapa Distr.: 1 O', 1 ㅇ (CP), Bol'shoy Utrish Vil., 1-5.05.2008, E.A. Khachikov; 1 + (CP), Bol'shoy Utrish Vil., Vodopadnaya Shchel', Quercus \& Carpinus wood, 10.06. 2009, A.V. Ponomarev; 1 (CP), same place, Lobanova Shchel', 06.2006, K.B. Gongal'skiy. North Osetiya Republic: Alagir Distr.: 1 ( (CP), 1 km N Tsrau Vil., 650 m, 15.07.2011, Yu.E. Komarov. Ardon Distr.: 1 (CP), 1 km S Bekan Vil., Bekan water reservoir, 530 m, 21.07.2011, Yu.E. Komarov. Rostov Area: Neklinovskiy Distr.: $1 O^{7}$ (CP), Morskoy Chulek Vil., 06.2010, A.A. Kondakov. Rostov-on-Don: $1 \mathrm{O}^{7}(\mathrm{CP})$, Botanical Garden of Rostov State University, 05.2003, Tsybelko. Tsymlyansk Distr.: $3 \sigma^{7} \sigma^{7}$ 5 우 (CP), without an exact location, 06.1971, A.V. Ponomarev. Ust-Donetsk Distr.: $10^{7}$ (CP), Razdorskaya Vil., Atamanskaya
Valley, woodland plantation, 20.05.2000, A.V. Ponomarev; $110^{7} \mathrm{O}^{7}$, 3 우 (CP), same place, meadow and woodland plantation, 17.0519.07.2001, A.V. Ponomarev; $2 \sigma^{7} \sigma^{7}(\mathrm{CP})$, vicinity of Razdorskaya Vil., Pukhlyakovskie Sklony, meadow, 15-19.05.2003, A.V. Ponomarev; $2 \sigma^{7} \sigma^{7}, 4$ 우 (CP), Krymskiy Vil., Savateevskaya Valley, pitfall traps, 3.06-31.07.2006, A.V. Ponomarev. Volgograd Area: $1+$ (CP), vicinity of Kamyshin Vil., 12-13.06.2005, E.A. Belosludtsev; 2 오 (CP), 6 km NW Trekhostrovskiy Vil., 'Donskoy' Natural Park, wood edge, pitfall traps, 6-15.07.2011, A.S. Khnykin. UKRAINE. The Crimea: Bakhchisaray Distr.: 1 (TNU), Kudrino Vil., 2.09.1995, M.M. Kovblyuk; 3 우 (TNU-1201/1/2), Crimean State Nature Reserve, ca. $2 \mathrm{~km} \mathrm{NW}$ of Zelenyi Gai kordon, bank of Alma River, 15.06.2000, M.M. Kovblyuk; 1 क (TNU1039/1), Crimean State Nature Reserve, Tar'er kordon, 30.06.2001, M.M. Kovblyuk; 1 + (TNU-2567/6), vicinity of Krasnozor'e Vil., 11.05.2008, A.A. Nadolny. Belogorsk Distr.: 1 (TNU), 7 km S of Zuya Vil., vicinity of Balanovo water reservoir on the Zuya River, 3.05.1996, M.M. Kovblyuk. Feodosiya Distr.: $13 \sigma^{7} \sigma^{7}, 22$ 우 (TNU$1762 / 1,1764 / 6,1765 / 1,1767 / 2,1972 / 1 / 2,1989 / 1,2028 / 1,2038 /$ $1,2039 / 1 / 2,2370 / 1,2380 / 1,2381 / 2,2382 / 1,2383 / 1 / 2,2384 / 1$, $2556 / 1,2559 / 1,2593 / 1 / 2,2602 / 1,2609 / 1,2610 / 1 / 2,2617 / 1,2649 /$ 

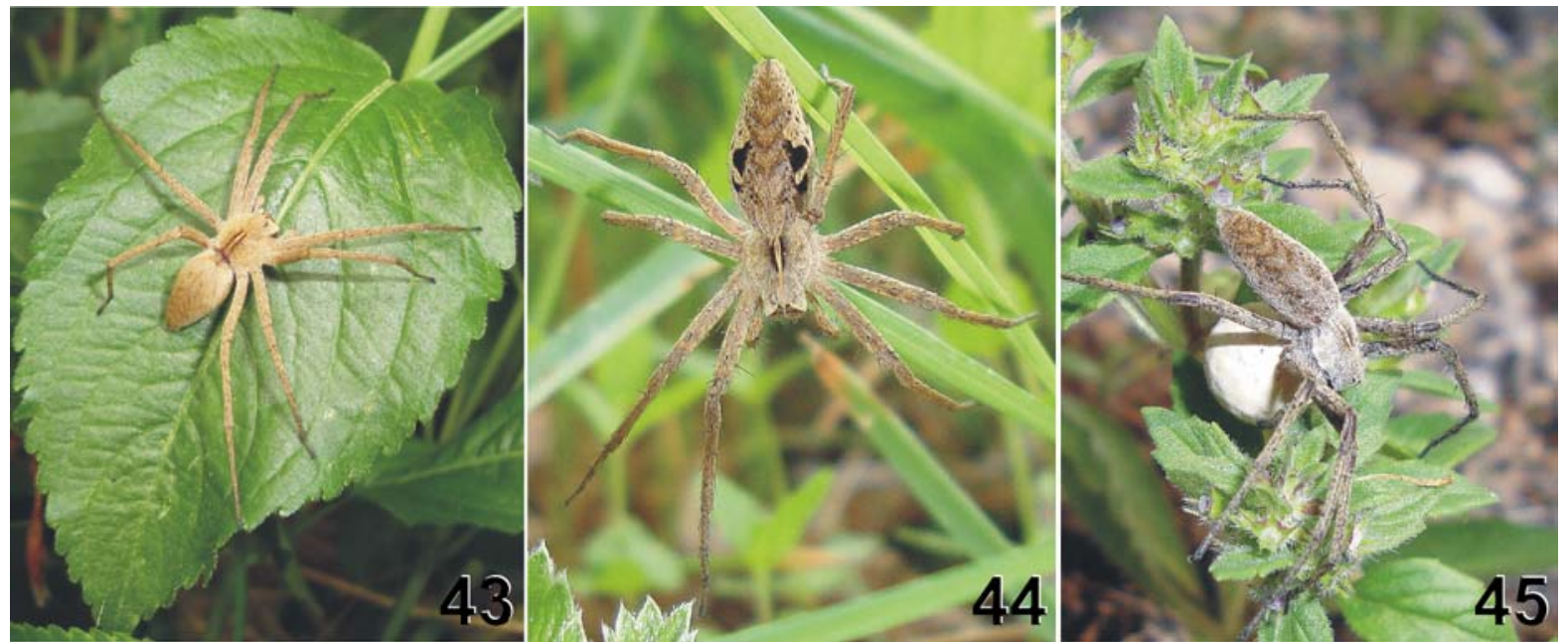

Figs 43-45. Females of Pisaura novicia in natural habitat (the Crimea, Dolgorukovskaya Yaila Mt. Range).

Рис. 43-45. Самки Pisaura novicia в естественной среде обитания (Крым, Долгоруковская яйла).

1, 2650/1, 2735/2, 2737/5/1), Karadag Nature Reserve, by hand picking and pitfall traps, 26.05.2003-2.06.2008, M.M. Kovblyuk, O.V. Kukushkin, A.A. Nadolny. Sevastopol Distr.: $1{ }^{\top}, 1$ (TNU), Chernaya River Canyon, 23.05.2000, O.V. Kukushkin; 2 90 (TNU1973/1), vicinity of Kolkhoznoe (Uzundzha) Vil., Uzundzha River Valley, 6.06.2005, O.V. Kukushkin; 1 ㅇ (TNU-2289/1), 3 km E Balaklava Vil., Kayu Mt. Rang, 14.06.2006, O.V. Kukushkin. Simferopol Distr.: 1 q (TNU), Simferopol, 23.05.1996, V.G. Stepanov \& A.V. Shumskiy; $1 O^{7}, 1$ (TNU), vicinity of Krasnoles'e Vil., 8.06. \& 19.06.1997, M.M. Kovblyuk; 1 (TNU), 2 km N of Pionerskoe Vil., 10.06.1998, M.M. Kovblyuk; 1 (TNU), vicinity of Lozovoe Vil., 10-13.07.1999, V.G. Stepanov; $1 \sigma^{7}$ (TNU), Simferopol, 3.05.2000, A.V. Shumskyi; $3 \sigma^{7} \sigma^{7}, 2$ 우 (TNU-X19, X20, X23), vicinity of Simferopol water reservoir, Bairakly Mt., S slope,

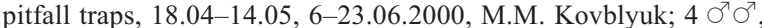
3 우 (TNU-1863/5, 1864/3, 1910/1, 1911/1), Chatirdag Mt. Rang, NE slope, pitfall traps, 7.05-1.06.2000, M.M. Kovblyuk; 2 우 (TNU-2173/1), vicinity of Perevalnoe Vil., Krasnopeshcherskoe Vil., 12.05.2000, M.M. Kovblyuk; $3 \sigma^{\top} \sigma^{\top}$ (TNU), vicinity of Lozovoe Vil., Kessler Forest, pitfall traps, 27.05--6.06.2000, M.M. Kovblyuk; $9 \sigma^{\top} \sigma^{\top}, 2$ 우 (TNU), ca. $0.5 \mathrm{~km} \mathrm{~S}$ of Lozovoe Vil., pitfall traps, 1.05-23.06.2000, M.M. Kovblyuk; $6 \sigma^{\top} \sigma^{\top}, 1$ (TNU985/5, 986/1, 987/2), Simferopol water reservoir, Zmeinyi Bay, pitfall traps, 1.05-6.06.2000, M.M. Kovblyuk; $2 \sigma^{\top} \sigma^{7}, 1$ (TNU$1008 / 2,2216 / 1), 1.5 \mathrm{~km} \mathrm{~N}$ dam of Simferopol water reservoir, Bitak Mt., by hand picking and pitfall traps, 14-26.05.2000, 4.06.2001, M.M. Kovblyuk; $1 \sigma^{7}$ (TNU-2189/3), Simferopol, Petrovskie Skaly Mt., 23.04.2001, M.M. Kovblyuk; 1 (TNU-2213/1),

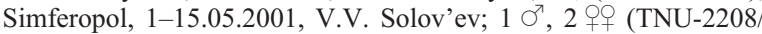
1), vicinity of Krasnoles'e Vil., Kosh-Kaya Mt. and Zantugai Valley, 21-30.06.2001, E.Yu. Sviridenko; 2 of (TNU-2105/1, 2110/1), vicinity of Krasnoles'e Vil., 22-23.06.2001, M.M. Kovblyuk; 3 ○ $\sigma^{\top}$ (TNU-1834/1, 1846/1), vicinity of Skvortsovo Vil., pitfall traps, 27.04-9.05. \& 19.05-1.06.2002, M.M. Kovblyuk; 1 ๆ (TNU-2056 1), vicinity of Perevalnoe Vil., Dolgorukovskaya Yaila Mt. Rang, near Bezdonnaya Cave, 14.05.2006, G.A. Prokopov. Sudak Distr. $1 \sigma^{7}$ (TNU), between Shelkovichnoe and Veseloe Vil., 26.05.1998, M.V. Onchurov; 9 ○ $^{\top}, 10$ 우 (TNU-2658/1, 2659/1, 2660/1, 2661/ 1, 2662/1, 2672/1, 2673/1, 2675/1, 2680/1, 2682/1, 2694/1, 2704/1, 2705/1), $10 \mathrm{~km}$ W Sudak, vicinity of Mezhdurech'e Vil., by hand picking and pitfall traps, 18.04-24.06.2010, M.K. Yusufova. Yalta Distr.: 6 O $^{7}, 6$ 우 (TNU-1106/4, 1112/2, 1113/4, 1141/4, 1142/4, 1143/4, 2341/6, 2352/1, 2354/1), Martyan Cape Reserve, pitfall traps, 7.05.2000-10.06.2007, M.M. Kovblyuk; $1 \sigma^{\top}$ (TNU-2452/1), same place, 20.04.2002, V.A. Gnelitsa; $1 \sigma^{7}$ (TNU-1377/7), Yalta Mountain-Forest Nature Reserve, ca. $1 \mathrm{~km} \mathrm{~N}$ of Nikita Vil., pitfall traps, 20-27.05.2000, M.M. Kovblyuk; $1 \sigma^{7}$ (TNU-2534/2), same place, pitfall traps, 05.2002, A.A. Khaustov; $2 \sigma^{\top} \sigma^{\top}, 2$ 우 (TNU1639/1, 2492/2, 2535/1), Yalta Mountain-Forest Nature Reserve, vicinity of Yalta, 27.05.-8.06.2002, M.M. Kovblyuk.

PUBLISHED FAUNISTIC RECORDS. Koch L. [1878]: Georgia (Borzhomi), Russia (Tuapse); Simon [1898, 1899]: the Caucasus, Abkhazia (Gagra), Georgia (Batumi, Zugdidi), Turkmenistan (Ashkhabad); Blandin [1976]: Uzbekistan (Bukhara); Brignoli [1984]: Italy, Israel; Logunov [1990: sub. P. mirabilis]: Russia (Belgorod Area, Krasnodar Area, North Osetiya), Azerbaijan (Zakatal'skiy Reserve), Kazakhstan (Dzhanybek), Turkmenistan (Syunt-Khasardinskiy Reserve); Mcheidze [1997]: Georgia (Manglisi, Poti); Pkhakadze [2006]: Georgia (Tbilisi) — quoted after Otto \& Dietzold [2006]; Kovblyuk et al. [2011]: Abkhazia (Bzyb' Vil., Gagra, nr. Pitsunda, kordon Tsymur).

COMMENTS. Brignoli [1984] recorded and illustrated $P$. novicia from Italy and Israel, but his and our illustrations of $P$. novicia are not identical. In his paper [Brignoli, 1984: figs 3-4], the epigyne has the narrow septum and the wide copulatory openings, but our specimens have the epigyne with the wide septum and the very narrow copulatory openings. The record of $P$. novicia by Brignoli [1984] should probably be assigned to $P$. mirabilis. Thus, some of the records of $P$. novicia from the Mediterranean are likely to be misidentifications. Brignoli also recorded $P$. orientalis, another poorly-known Pisaura species, from Italy, Greece and Lebanon. Male of this species remains unknown, while the description of the female is not satisfactory. $P$. orientalis was described from Waluyki (Belgorod Area, Russia) [Kulczyński, 1913]. There are specimens of $P$. novicia from Belgorod Area in our material. Logunov also recorded $P$. novicia from Belgorod Area [Logunov, 1990: sub. P. mirabilis]. Therefore, it is likely that the name $P$. orientalis is a junior synonym of $P$. novicia. The records of $P$. orientalis from the Medi- 


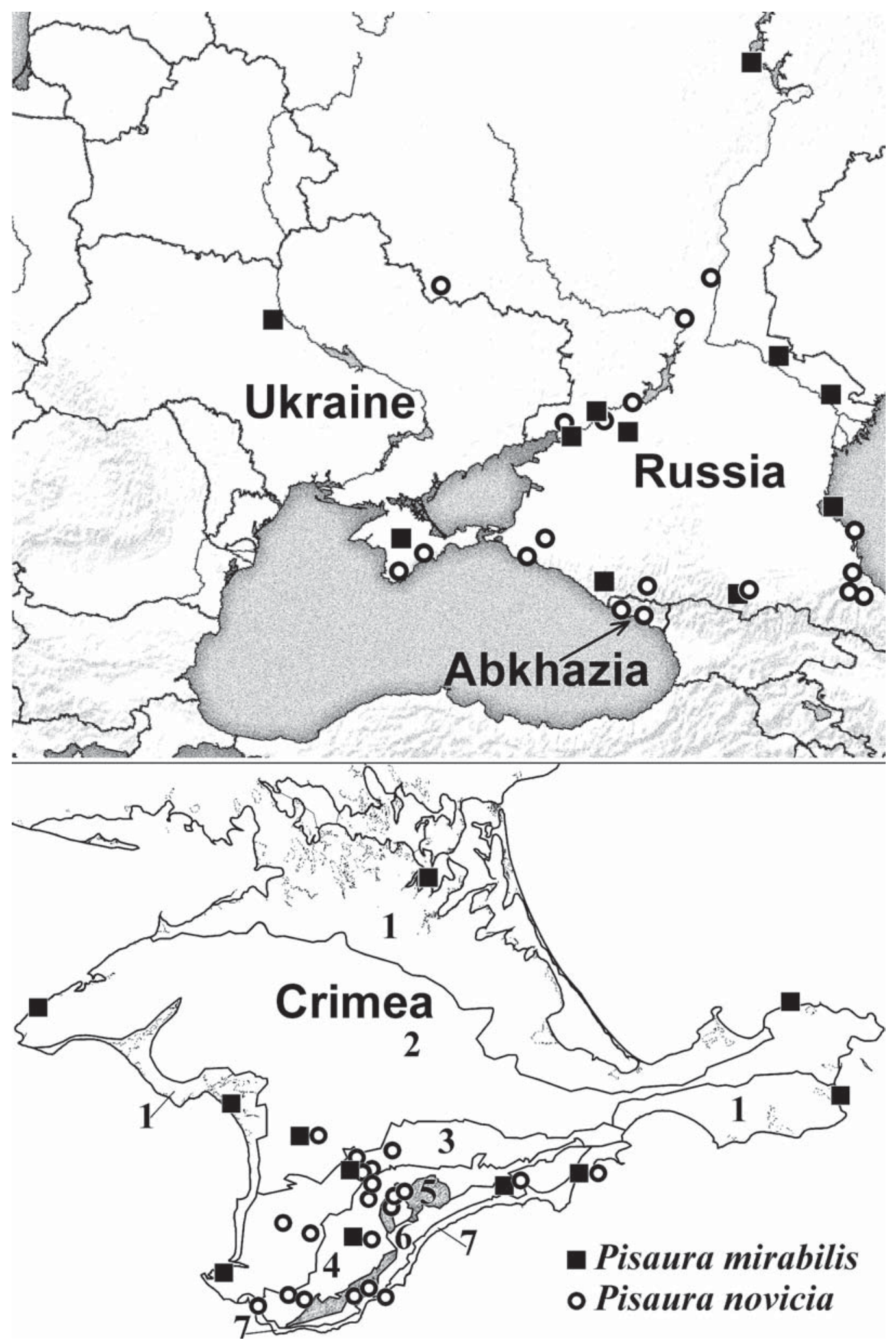

Map 1. Distribution of Pisaura mirabilis and P. novicia based on the studied specimens (1-7 - landscape zones of the Crimea, see explanations in the text).

Карта 1. Распространение Pisaura mirabilis и P. novicia, по материалам наших коллекций (1-7 — ландшафтные зоны Крыма, пояснения в тексте). 
terranean need confirmation upon reference to the pertinent material. Also, $P$. novicia is recorded from Uzbekistan [Blandin, 1976] and Turkmenistan [Simon, 1899; Logunov, 1990: sub. P. mirabilis]. Logunov supported all his records of $P$. mirabilis from Turkmenistan and Russia (Belgorod Area, Krasnodar Area, North Osetiya), Azerbaijan and Kazakhstan by drawings of the copulatory organs of studied specimens [Logunov, 1990: pl. 2, f. в, г, д, e, ж, з; pl. 3, f. II $a$, IIб]. His illustrations leave no doubt that he actually dealt with $P$. novicia. Simon [1898, 1899] did not provide any drawing in his papers. The illustration of the epigyne of $P$. novicia given by Blandin [1976] cannot be used for species identification. Therefore, records both from Uzbekistan and from Turkmenistan are doubtful. The distribution of $P$. novicia based on the studied specimens is shown in Map 1.

DIAGNOSIS. Females of three species - P. acoreensis, $P$. consocia and $P$. quadrilineata - are similar to those of $P$. novicia. The septum of the epigyne in $P$. novicia, $P$. acoreensis, $P$. consocia and $P$. quadrilineata is wide (cf. Figs 28-30, 33, 40-42) [Wunderlich, 1992: 658, 662], unlike females of other Pisaura species having the narrow epigynal septum. The female of $P$. novicia differs from that of $P$. consocia by the absence of septal ridge (longitudinal ridge on the septum in P. consocia) (cf. Figs 28-30, 33, 40-42 and Brignoli, 1984: fig. 16; Levy, 1999: fig. 32A]. The females of $P$. novicia, $P$. acoreensis and $P$. quadrilineata are difficult to distinguish. The males of $P$. novicia are similar to those of $P$. mirabilis (see above under 'Diagnosis' for P. mirabilis).

DESCRIPTION. Male and female from the Crimea. Measurements (male/female): total length 10.5 / 11.5; carapace 4.8 / 4.4 long, 3.8 / 3.5 wide. Length of palp segments (male/female): femur $2.0 / 1.7$, patella 0.9 / 0.8 , tibia $0.7 / 1.0$, tarsus $2.2 / 2.0$. Length of leg segments (male/female):

\begin{tabular}{|l|l|l|l|l|l|}
\hline Leg & Femur & Patella & Tibia & Metatarsus & Tarsus \\
\hline I & $5.2 / 5.0$ & $1.9 / 1.9$ & $5.2 / 4.9$ & $5.0 / 4.5$ & $2.4 / 2.0$ \\
\hline II & $5.4 / 5.2$ & $1.9 / 1.8$ & $5.1 / 4.9$ & $5.0 / 4.7$ & $2.5 / 2.1$ \\
\hline III & $4.6 / 4.6$ & $1.7 / 1.6$ & $4.0 / 4.0$ & $3.9 / 4.0$ & $1.7 / 1.6$ \\
\hline IV & $5.7 / 5.8$ & $1.7 / 1.7$ & $5.3 / 5.2$ & $6.0 / 6.0$ & $2.3 / 2.1$ \\
\hline
\end{tabular}

Male leg spination.

\begin{tabular}{|c|c|c|c|c|}
\hline & Femur & Patella & Tibia & Metatarsus \\
\hline Leg I & $\begin{array}{l}\text { d } 1-1-1 \text { (a), } \\
\text { pl } 1-1-1, \text { rl } \\
1-1-1-1-1\end{array}$ & d 1 (a) & $\begin{array}{l}\text { d 1-1, pl 1- } \\
1, \text { rl 1-1, v } \\
2-2-2-2 \text { (a) }\end{array}$ & $\begin{array}{l}\text { pl } 1-1-1 \text { (a), } \\
\text { rl 1-1-1 (a), v } \\
2-2-3 \text { (a) }\end{array}$ \\
\hline Leg II & $\begin{array}{l}\text { d 1-1-1 (a), } \\
\text { pl } 1-1-1-1- \\
1 \text {, rl 1-1-1- } \\
1-1\end{array}$ & d 1 (a) & $\begin{array}{l}\text { d 1-1, pl 1- } \\
1, \text { rl 1-1, v } \\
2-2-2-2 \text { (a) }\end{array}$ & $\begin{array}{l}\text { pl } 1-1-1 \text { (a), } \\
\text { rl } 1-1-1(a), v \\
2-2-3 \text { (a) }\end{array}$ \\
\hline Leg III & $\begin{array}{l}\text { d 1-1-1 (a), } \\
\text { pl } 1-1-1-1- \\
1, \text { rl 1-1-1- } \\
1-1\end{array}$ & d 1 (a) & $\begin{array}{l}\text { d 1-1, pl 1- } \\
1, \text { rl 1-1, v } \\
2-2-2 \text { (a) }\end{array}$ & $\begin{array}{l}\text { pl 1-1-1 (a), } \\
\text { rl 1-1-1 (a), v } \\
2-2-3 \text { (a) }\end{array}$ \\
\hline Leg IV & $\begin{array}{l}\text { d } 1-1-1 \text { (a), } \\
\text { pl } 1-1-1-1, \\
\text { rl 1-1 }\end{array}$ & d 1 (a) & $\begin{array}{l}\text { d } 1-1, \text { pl } 1- \\
1, \text { rl 1-1, v } \\
2-2-2 \text { (a) }\end{array}$ & $\begin{array}{l}\text { pl 1-1-1 (a), } \\
\text { rl 1-1-1 (a), v } \\
2-2-3 \text { (a) }\end{array}$ \\
\hline
\end{tabular}

Male palp spination. Femur: d 1-1 (a), pl 1; patella: d 1-1 (a), pl 1; tibia: d 1, pl 2; cymbium: pl 2-1, v 2.
Female leg spination.

\begin{tabular}{|c|c|c|c|c|}
\hline & Femur & Patella & Tibia & Metatarsus \\
\hline Leg I & $\begin{array}{l}\text { d } 1-1-1(a), \\
\text { pl } 1-1-1-1 \text {, } \\
\text { rl 1-1-1-1-1 }\end{array}$ & d 1 (a) & $\begin{array}{l}\text { d 1-1, pl 1- } \\
1, \text { rl 1-1, V } \\
2-2-2-2 \text { (a) }\end{array}$ & $\begin{array}{l}\text { pl 1-1-1 (a), } \\
\text { rl 1-1-1 (a), v } \\
2-2-3 \text { (a) }\end{array}$ \\
\hline Leg II & $\begin{array}{l}\text { d } 1-1-1 \text { (a), } \\
\text { pl 1-1-1-1- } \\
1 \text {, rl 1-1-1- } \\
1-1\end{array}$ & d 1 (a) & $\begin{array}{l}\text { d 1-1, pl 1- } \\
1, \text { rl 1-1, v } \\
2-2-2-2 \text { (a) }\end{array}$ & $\begin{array}{l}\text { pl } 1-1-1 \text { (a), } \\
\text { rl 1-1-1 (a), v } \\
2-2-3 \text { (a) }\end{array}$ \\
\hline Leg III & $\begin{array}{l}\text { d } 1-1-1 \text { (a), } \\
\text { pl } 1-1-1-1- \\
1, \text { rl 1-1-1- } \\
1-1\end{array}$ & d 1 (a) & $\begin{array}{l}\text { d 1-1, pl 1- } \\
1, \text { rl 1-1, v } \\
2-2-2 \text { (a) }\end{array}$ & $\begin{array}{l}\text { pl } 1-1-1 \text { (a), } \\
\text { rl 1-1-1 (a), v } \\
2-2-3 \text { (a) }\end{array}$ \\
\hline Leg IV & $\begin{array}{l}\text { d } 1-1-1(a), \\
\text { pl } 1-1-1-1, \\
\text { rl } 1-1\end{array}$ & d 1 (a) & $\begin{array}{l}\text { d 1-1, pl 1- } \\
1, \text { rl 1-1, v } \\
2-2-2 \text { (a) }\end{array}$ & $\begin{array}{l}\text { pl 1-1-1 (a), } \\
\text { rl 1-1-1 (a), v } \\
2-2-3 \text { (a) }\end{array}$ \\
\hline
\end{tabular}

Female palp spination. Femur: d 1-1 (a), pl 2; patella: d 1-1 (a), pl 1; tibia: d 1-1 (a), pl 2; tarsus: pl 2-1, rl $1, \mathrm{v} 2$.

Male copulatory organs: Figs 2, 4, 6, 8, 12-14, 1718, 20-21, 24-25, 36-38. As P. novicia and P. mirabilis have a similar morphology of the copulatory organs (see above under $P$. mirabilis for the nomenclature of sclerites and further explanations).

Female copulatory organs: Figs 28-30, 33-34, 4042. As $P$. novicia and $P$. mirabilis have a similar morphology of the copulatory organs (see above under $P$. mirabilis for the nomenclature of sclerites and further explanations).

VARIATION. Male $(\mathrm{n}=6)$ : carapace 3.6-5.0 long, 3.0-4.0 wide; female $(n=5)$ : carapace $4.0-4.8$ long, $3.0-4.0$ wide. Some females with black spots on the dorso-lateral side of abdomen (Fig. 44).

TYPE LOCALITY. L. Koch [1878] described $P$. novicia on the basis of several females (syntypes) collected from Borzhomi (Georgia) and Tuapse (Krasnodar Area, Russia).

DISTRIBUTION. East Mediterranean-Central Asian temperate-subtropical range: Ukraine (the Crimea), Russia (Belgorod Area, Dagestan Republic, Karachaevo-Cherkesiya Republic, Krasnodar Area, Rostov Area, North Osetiya Republic, Volgograd Area), Abkhazia, Georgia, Azerbaijan (Zakatal'skiy Reserve), Kazakhstan (Dzhanybek), Turkmenistan (SyuntKhasardaghsky Reserve) [Koch, 1878; Simon, 1898, 1899; Logunov, 1990: sub. P. mirabilis; Mcheidze, 1997; Otto \& Dietzold, 2006; Kovblyuk et al., 2011; present data]. All the records of $P$. novicia from Russia, except for Belgorod Area, Krasnodar Area and North Osetiya, have been made for the first time. $P$. novicia is recorded from the Crimea and Ukraine for the first time.

PHENOLOGY. In the Crimea $\bigcirc^{\top} \sigma^{\top}-$ IV-VI,, $0+$ IV-VII (single female at IX), peak in May. Females with egg sacs - V-VI.

\section{Discussion}

In Ukraine (Crimea) and Russia (Rostov Area, North Osetiya), $P$. mirabilis and $P$. novicia show a sympatric 
distribution and even co-habitation. Yet, no differences have been found in their phenology. However, both species are easily distinguishable by their copulatory organs (see above) and thus there is no doubt that both can co-exist in the same habitats. P. mirabilis and $P$. novicia are sympatric all over Crimea, apart from two landscape zones: 1) the mountain meadows and steppes (800-1500 m a.s.1.) where $P$. mirabilis is absent; 2) the semi-desert steppe and saline lands ( $0-40 \mathrm{~m}$ a.s.1.) where $P$. novicia is absent. The sympatric co-existence of these species is uncommon and requires a further, detailed study.

Specimens of $P$. mirabilis and $P$. novicia were found in all Crimean landscape (physical-geographic) zones. There are seven major landscape zones in the Crimea: 1 - semi-desert steppe and saline lands; 2 - true steppe; 3 - premontane forest steppe; 4 forests of the northern slope of Crimean mountains; 5 - mountain meadows and steppes; 6 - forests of the southern slope of Crimean mountains; 7 - subMediterranean vegetation of the Southern Coast of Crimea [Biodiversity ..., 1999]. The landscape distribution of $P$. mirabilis and $P$. novicia is shown in Table 1 and Map 1.

ACKNOWLEGENS. Authors sincerely thank all the collectors who provided specimens for this study: S.V. Alieva, Yu.G. Arzanov, E.A. Belosludtsev, V.A. Gnelitsa,
Table 1. Distribution of $P$. mirabilis and $P$. novicia in the landscape zones of the Crimea. Таблица 1. Ландшафтное распространение P. mirabilis и P. novicia в Крыму.

\begin{tabular}{|l|c|c|}
\hline Landscape zone & P. mirabilis & P. novicia \\
\hline $\begin{array}{l}\text { 1. Semi-desert steppe and saline } \\
\text { lands }\end{array}$ & + & - \\
\hline 2. True steppe & + & + \\
\hline 3. Premontane forest steppe & + & + \\
\hline 4. Forests of the northern slope & + & + \\
\hline $\begin{array}{l}\text { 5. Mountain meadows and } \\
\text { steppes (yaila) }\end{array}$ & - & + \\
\hline 6. Forests of the southern slope & + & + \\
\hline $\begin{array}{l}\text { 7. Sub-Mediterranean area of the } \\
\text { southern coast }\end{array}$ & + & + \\
\hline
\end{tabular}

K.B. Gongal'skiy, V.P. Isaev, P.P. Ivliev, E.A. Khachikov, A.Kh. Khalidov, A.A. Khaustov, A.S. Khnykin, V.F. Kobzar', Yu.E. Komarov, A.A. Kondakov, O.V. Kukushkin, A.S. Matyukhin, M.V. Onchurov, G.A. Prokopov, A.V. Shumskiy, V.V. Solov'ev, V.G. Stepanov, I. Strachova, E.Yu. Sviridenko, A.S. Tili, Tsybelko, A.A. Zotov, D.D. Volkova, N.N. Yunakov, M.K. Yusufova, V.K. Zykova. We thank P.E. Gol'din (Simferopol) for improving the English of the earlier draft. Authors are grateful to R. Leech (Edmonton) who kindly checked English of the final draft. Also, we wish to thank D.V. Logunov and T. Blick for providing us with some references and D.V. Logunov for improving manuscript. This work of M.K. was supported by the Karadag Nature Reserve.

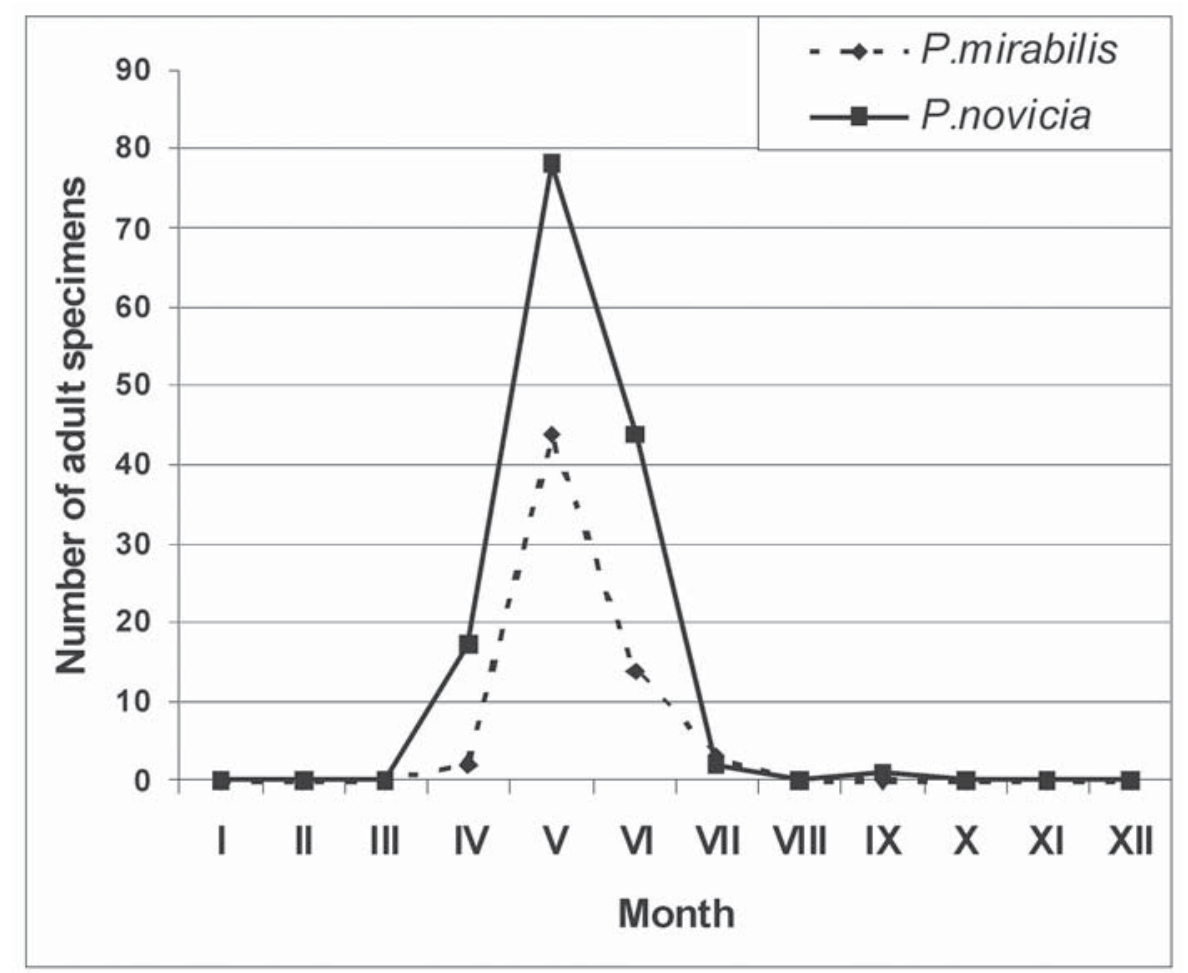

Fig. 46. Comparative phenology of Pisaura mirabilis and P. novicia based on the adult specimens collected in the Crimea. Рис. 46. Сравнение фенологии взрослых особей Pisaura mirabilis и P. novicia, собранных в Крыму. 


\section{References}

Almquist S. 2005. Swedish Araneae, part 1 - families Atypidae to Hahniidae (Linyphiidae excluded) // Insect Systematics and Evolution. Supplement No.62. P.1-284.

Biodiversity Support Program. Priority-setting in Conservation: A new approach for Crimea. 1999. Results of the Conservation Needs Assessment in Crimea. Washington: BSP. 257 p. [in Russian and English].

Blandin P. 1976. Etudes sur les Pisauridae africaines VI. Definition des genres Pisaura Simon, 1885, Pisaurellus Roewer, 1961, Afropisaura n. gen. et mise au point sur les especes des genres Afropisaura and Pisaurellus (Araneae Pisauridae Pisaurinae) // Revue Zool. afr. Vol.90. No.4. P.917-939.

Brignoli P.M. 1984. Zur Problematik der mediterranen PisauraArten (Arachnida, Araneae, Pisauridae) // Zool. Anz. Bd.213. Nr.1/2. S.33-43.

Clerck C. 1757. Svenska spindlar. Aranei Svecici. Stockholmiae. $16+154 \mathrm{p}$.

Gorodkov K.B. 1984. [Types of ranges of insects inhabiting tundra and taiga zones of European part of USSR] // Arealy nasekomykh Evropeiskoi chasti SSSR, Karty 179-221. Leningrad: Nauka Press. P.3-20 [in Russian].

Harvey P.R., Nellist D.R., Telfer M.G. (eds.). 2002. Provisional atlas of British spiders (Arachnida, Araneae). Vol.1-2 / Huntington: Biological Records Centre. 406 p.

Helsdingen P., van. 2011. Fauna Europaea: Araneae. Fauna Europaea version 2.4. online at: http://www.faunaeur.org

Koch L. 1878. Kaukasische Arachnoiden // Schneider O. (ed.) Naturwissenschaftliche Beitrage zur Kenntniss der Kaukasuslander. Dresden: Verlag der Burdach'schen Hofbuchhandlung. S.36-71.

Kovblyuk M.M., Marusik Yu.M., Ponomarev A.V., Gnelitsa V.A., Nadolny A.A. 2011. Spiders (Arachnida: Aranei) of Abkhazia // Arthropoda Selecta. Vol.20. No.1. P.21-56.

Kulczyński V. 1913. Arachnoidea // V. Velitchkovsky. Faune du district de Walouyki du gouvernement de Woronege (Russie). Cracovie. Fasc.10. P.1-30.

Levy G. 1999. The lynx and nursery-web spider families in Israel (Araneae, Oxyopidae and Pisauridae) // Zoosystema. T.21. No.1. P.26-64.

Logunov D.V. 1990. [New data on spiders of the families Atypidae, Pisauridae and Thomisidae of the USSR fauna] // Zolotarenko G.S. (ed.). Fauna Sibiri. Chlenistonogie i gel'minty.
Novosibirsk: Biologicheskiy institut SO AN SSSR. P.33-43 [in Russian]

Marusik Yu.M., Kovblyuk M.M., Koponen S. 2011. A survey of the East Palaearctic Lycosidae (Araneae). 9. Genus Xerolycosa Dahl, 1908 (Evippinae) // Zookeys. No.119. P.11-27.

Mkheidze T.S. 1997. [Spiders of Georgia (taxonomy, ecology, zoogeographical review)]. Tbilisi: Tbilisi university. 390 pp. [in Georgian].

Otto S., Dietzold S. 2006. Caucasian Spiders. A faunistic database on the spiders of the Caucasus. Version 1.3. Online at: http:// caucasus-spiders.info

Patel S.K. 1987. A new spider species: Pisaura swamii sp. nov. (Pisauridae) // Biological Bulletin of India. Vol.9. No.1. P.6466.

Patel B.H., Reddy T.S. 1990. Two new species of the genus Pisaura Simon (Araneae: Pisauridae) from coastal Andhra Pradesh, India // Entomon. Vol.15. No.1-2. P.37-40.

Platnick N.I. 2012. The world spider catalog, version 12.5. American Museum of Natural History, online at: http://research.amnh. org/entomology/spiders/catalog/index.html

Pkhakadze V. 2006. [The spiders of Tbilisi valley (Arthropoda, Araneae): fauna, ecology, zoogeography]. PhD dissertation at the Dshavakhishvili University. 153 pp. [in Georgian] (Non Visum, cited after Otto \& Dietzold, 2006).

Sierwald P. 1989. Morphology and ontogeny of female copulatory organs in American Pisauridae, with special reference to homologous features (Arachnida: Araneae) // Smithsonian contributions to zoology. No.484. P.1-24.

Simon E. 1898. Histoire naturelle des araignees. T.2. Paris. P.193380 .

Simon E. 1899. Araneae Caucasicae // Radde G.I. Kollektsii Kavkazskogo Muzeya (Museum Caucasicum). T.1. Zoologiya. Tiflis: Tipographiya Kancelyarii Glavnonachalstvuyushchego grazhdanskoyu chastiyu na Kavkaze. P.478-480.

Wunderlich J. 1987. The spiders of the Canary Islands and Madeira: Adaptive Radiation, Biogeography, Revisions and Descriptions of New Species.Langen: Triops Verlag. $435 \mathrm{~S}$.

Wunderlich J. 1992. Die Spinnen-Fauna der Makaronesischen Inseln: Taxonomie, Ekologie, Biogeographie und Evolution // Beiträge zur Araneologie. Bd.1.619 S.

Zhang J.-X., Zhu M.-Sh., Song D.-X. 2004. A review of the Chinese nursery-web spiders (Araneae, Pisauridae) // The Journal of Arachnology. Vol.32. P.353-417.

Responsible editor D.V. Logunov 